Gut and Liver, Vol. 12, No. 2, March 2018, pp. 149-157

\title{
Efficacy of a Three-Dimensional-Printed Training Simulator for Endoscopic Biopsy in the Stomach
}

\author{
Sunpyo Lee ${ }^{1}$, Ji Yong Ahn ${ }^{1}$, Minkyu Han ${ }^{2}$, Gin Hyug Lee ${ }^{1}$, Hee Kyong $\mathrm{Na}^{1}$, Kee Wook Jung ${ }^{1}$, Jeong Hoon Lee ${ }^{1}$, Do Hoon
} $\mathrm{Kim}^{1}$, Kee Don Choi ${ }^{1}$, Ho June Song ${ }^{1}$, and Hwoon-Yong Jung ${ }^{1}$

Departments of ${ }^{1}$ Gastroenterology and ${ }^{2}$ Clinical Epidemiology and Biostatistics, Asan Medical Center, University of Ulsan College of Medicine, Seoul, Korea

Background/Aims: We used three-dimensional (3D) printing technology to create a new biopsy simulator for the stomach and investigated its efficacy and realism in endoscopic biopsy training. Methods: A novel stomach biopsy simulator, with 10 biopsy sites, was produced using a 3D printer. We enrolled 26 participants, including 10 residents, six firstyear fellows, five second-year fellows, and five faculty members. We recorded and reviewed five training sessions and evaluated the simulator with questionnaires using a 7-point Likert scale. Results: The mean completion time (seconds) was $244.8 \pm 11.5$ for the residents, $107.9 \pm 33.4$ for the firstyear fellows, $106.8 \pm 20.1$ for the second-year fellows, and $103.8 \pm 19.2$ for the faculty members. The completion time became shorter with repetition and was significantly lower for residents by the fifth trial (first trial, 347.0 \pm 159.5 ; fifth trial, 169.6 $\pm 57.7 ; p=0.007)$. The faculty members strongly agreed that the simulator realistically reflected endoscopic handling and was reasonable for endoscopic training (scores of $6.2 \pm 0.8$ and $6.4 \pm 0.9$, respectively). Importantly, experienced endoscopists reported that the difficulty levels of the 10 biopsy sites in the simulator were a realistic match for the actual stomach. Conclusions: This endoscopic biopsy simulator created using a 3D printer is a realistic and useful method to improve the biopsy skills of trainee endoscopists. (Gut Liver 2018;12:149-157)

Key Words: Printing, three-dimensional; Endoscopy; Simulator; Biopsy; Education

\section{INTRODUCTION}

Esophagogastroduodenoscopy (EGD) is an excellent diagnostic modality for various diseases, and performing a biopsy is an important endoscopic step for making an accurate diagnosis. Endoscopic diagnoses consist of detecting abnormal lesions and obtaining tissue samples via forceps. These methods are fundamental to the training of endoscopists who want to develop their skills for therapeutic procedures. Traditional endoscopic training usually involves supervised hands-on training with patients in the clinical setting, but this is considered to be risky for patients and thus requires them to volunteer. ${ }^{1,2}$ To replace this method, various endoscopic simulators have been developed. However, each of these systems has shown some limitations. Tissue-based simulators require special endoscopes for animal use, animal organ procurement, extensive preparation and disposal processes. Further, computerized virtual simulators are very costly and often inconveniently located away from the clinical activity areas. ${ }^{3}$ To overcome these limitations, our center previously developed an easy-to-manufacture and lowcost simulator using common materials such as plastic containers and snap fasteners; this simulator was found to be a useful, effective, and realistic method for improving biopsy techniques for novices. ${ }^{4}$

Recently, three-dimensional (3D) printing has become more accessible and has generated much interest in various fields of medicine. These include anatomy education and clinical disciplines such as in orthopedics, spinal surgery, neurosurgery, and cardiac surgery. Three-dimensional printing has become widely adopted in the medical field because 3D-printed materials can be used for practicing different procedures and thus to train stu-

\footnotetext{
Correspondence to: Gin Hyug Lee

Department of Gastroenterology, Asan Medical Center, University of Ulsan College of Medicine, 88 Olympic-ro 43-gil, Songpa-gu, Seoul 05505, Korea

Tel: +82-2-3010-3986, Fax: +82-2-2045-4043, E-mail: jhlee409@amc.seoul.kr

Received on March 15, 2017. Revised on May 10, 2017. Accepted on May 24, 2017. Published online October 27,2017

pISSN 1976-2283 eISSN 2005-1212 https://doi.org/10.5009/gnl17126

Sunpyo Lee and Ji Yong Ahn contributed equally to this work as first authors.

@ This is an Open Access article distributed under the terms of the Creative Commons Attribution Non-Commercial License (http://creativecommons.org/licenses/by-nc/4.0) which permits unrestricted non-commercial use, distribution, and reproduction in any medium, provided the original work is properly cited.
} 
dents. ${ }^{5-9}$ We applied 3D printing to the development of a biopsy simulator and thereby created a new simulator of the real stomach using a silicone reproduction. We investigated the efficacy and realism of this simulator for endoscopic biopsy trainees who have never performed an EGD. We compared the results from the trainees with those from more experienced endoscopists and experts and obtained the opinions of these participants on the utility of the simulator.

\section{MATERIALS AND METHODS}

\section{Participants}

Between January and July 2016, 26 subjects working at Asan Medical Center, Seoul, Korea participated in this study. Ten of these individuals were residents affiliated to the department of internal medicine with no EGD or colonoscopy experience. Six subjects were first-year clinical fellows, five were second-year clinical fellows, and five were medical faculties. All 26 participants were affiliated to the department of gastroenterology. All of the residents had completed training courses on endoscopic techniques for trainees, including the previously developed easy-to-manufacture simulator at our center. ${ }^{4}$ Prior to enrollment in this study, the median numbers of performed EGD procedures among the 16 study participants with experience were as follows: first-year fellows, 1,117 (interquartile range [IQR], 1,032 to 1,347); second-year fellows, 2,671 (IQR, 1,784 to 3,790); and medical faculties, 12,133 (IQR, 10,283 to 26,805). This study was approved by the Institutional Review Board of Asan Medical Center (IRB number: 2016-0302).

\section{Generation of an endoscopic biopsy simulator using a 3D printer}

To produce the silicone-based representation of the human stomach via 3D printing technology, the first step was to make
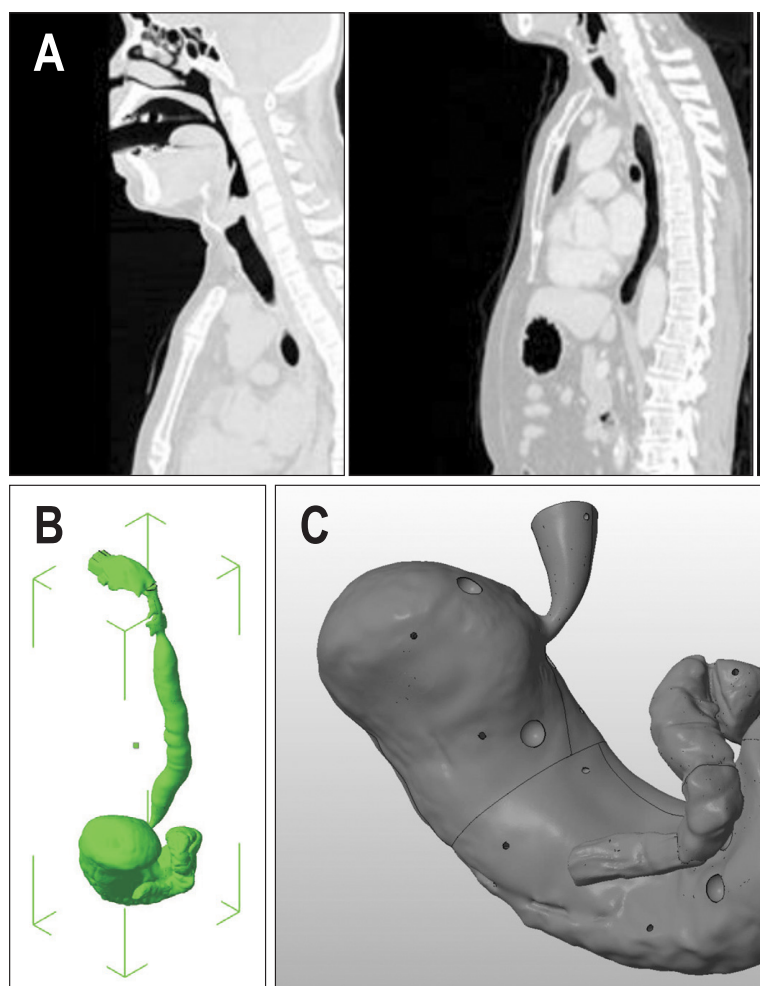

C
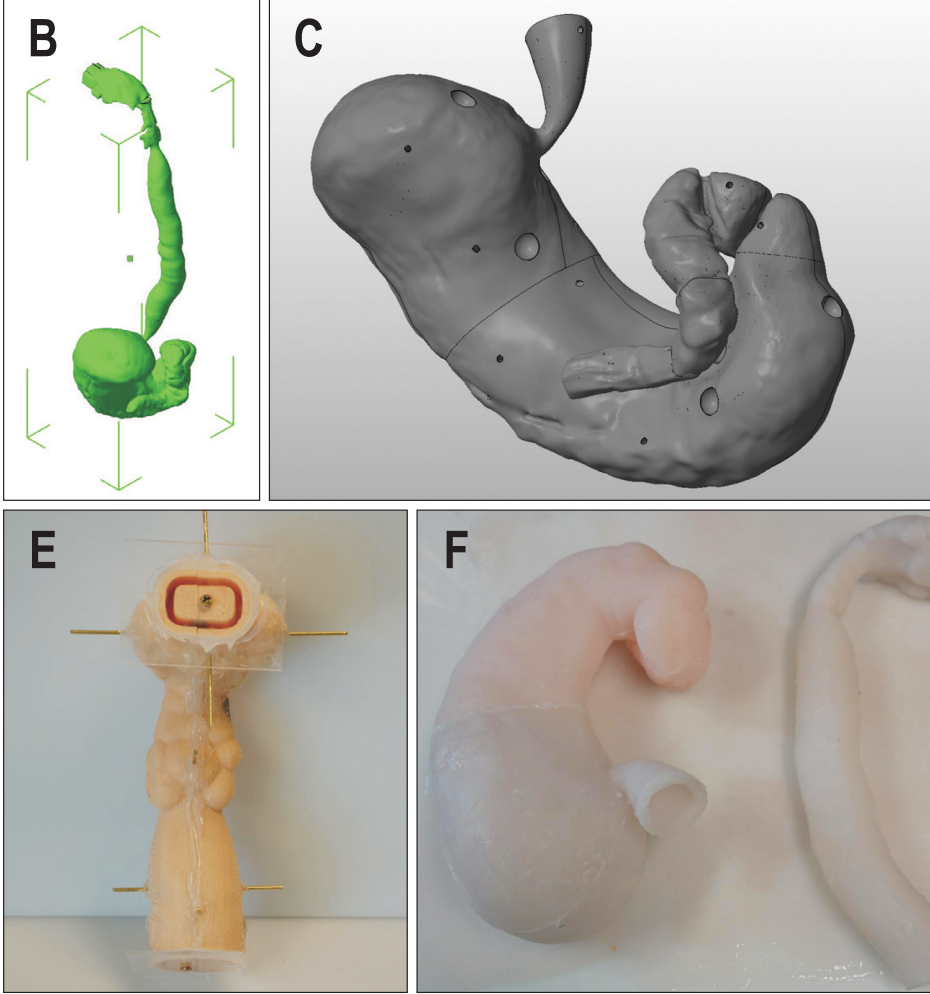

$\mathbf{F}$
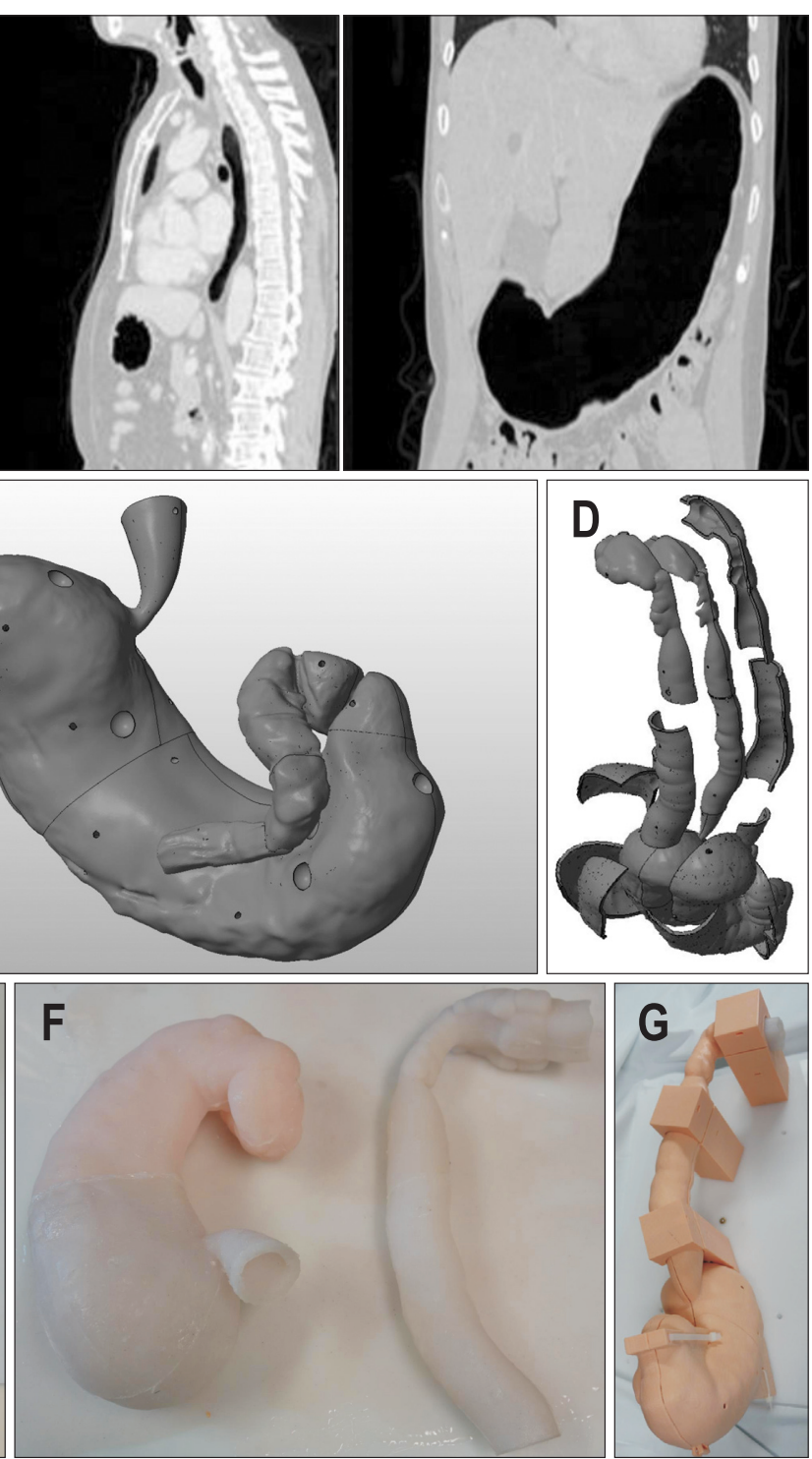

G

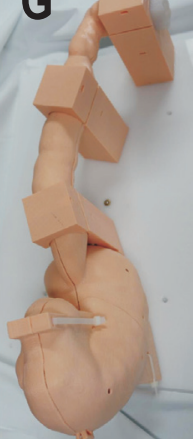

Fig. 1. (A) Representative images from a neck computed tomography (CT), chest CT, and CT gastrography. (B) Perspective view of the final upper gastrointestinal tract (UGIT) prototype. (C) Gastric section of the UGIT prototype with depressions for the gastric polyps. (D) Perspective view of the final shell and core of the UGIT silicone mold. (E) Front view of the upper part of the UGIT core-shell molding with all parts in position. (F) UGIT silicone moldings. For convenience, four UGIT silicone molding parts are fused with superglue into the gastric and upper sections. (G) Final assembled UGIT shell and UGIT silicone moldings. 
an upper gastrointestinal tract (UGIT) prototype. Digital Imaging and Communications in Medicine (DICOM) images from a neck computed tomography (CT), chest CT and CT gastrography from several anonymous subjects (Fig. 1A) were imported into the free open 3D modeling software, 3D slicer version 4.5.0 (www.slicer.org). ${ }^{10}$ The portion with air Hounsfield units from the mouth to the duodenal second portion was selectively segmented. This segmented portion was then reconstructed into the pre-UGIT prototype model and edited with a 3D model editing program, MeshMixer 3.0 (Autodesk, San Rafael, CA, USA). The final UGIT prototype model (Fig. 1B) was exported as an STL file.

The second step of the process was the UGIT silicone molding (the core and the shell). The UGIT prototype STL file was imported into a 3D model editing program, Netfabb professional version 5 (Netfabb GmbH, Lupburg, Germany). To simulate gastric polyps, 10 8-mm sized balls were made and subtracted from the gastric part of the UGIT prototype at the designated locations (Fig. 1C). The distance between the core outer surface and the shell inner surface was $6 \mathrm{~mm}$, and the thickness of the shell itself was $4 \mathrm{~mm}$. The final products were cut into several pieces which were printable at each $3 \mathrm{D}$ printer and convenient to detach the silicone from the molding wall, and exported as STL files for 3D printing (Fig. 1D). The STL files were then printed using a fused deposition modeling (FDM) 3D printer (clone S270 and clone K300, K.Clone, Daejeon, Korea; Replicator 2, MakerBot, Brooklyn, NY, USA) in a layer-by-layer manner. The settings of the 3D printers were adjusted in accordance with the manufacturer's instructions. The printed parts were assembled with a glue gun and transparent acryl plate (Fig. 1E). We used the PlatSil Gel-10 (Polytech, Easton, PA, USA) silicone kit, which is a platinum silicone rubber (Shore A10). The mixed silicone was injected through the hole into the space between the core and the shell using a silicone injection gun. The injected silicone was then cured at room temperature for 1 hour. Then the silicone moldings were fused with superglue into two parts (Fig. 1F).

The third step involved making the UGIT shell, which was a similar process to the shell production in the second step. The UGIT prototype STL file was imported into the 3D model editing program, Netfabb professional version 5 (Netfabb $\mathrm{GmbH}$ ). The thickness of the shell itself was $4 \mathrm{~mm}$. Several columns between the UGIT shell and the board were located on the board to support the UGIT shell. The STL files were printed with the FDM 3D printers and the polylactic acid filaments.

The fourth step was the assembly of the final E\&B simulator. The UGIT shell was attached to the board (acryl, $400 \times 550 \times 8$ $\mathrm{mm}$ ) with superglue. The final silicone molding was put into the UGIT shell. Parts of the UGIT shell were tightened with cable ties (Fig. 1G).

\section{Three-dimensional biopsy simulator training}

The EGD training course using the new simulator system consisted of the following steps: (1) the participants started the training session by placing the scope at the gastroesophageal junction; (2) the first lesion was approached; (3) a biopsy forceps was inserted through the procedure hole; (4) a biopsy sample was obtained from the lesion; (5) an assistant checked whether the fragmented silicone was contained in the forceps; and (6) the participant moved to the next lesion irrespective of the result. These steps were repeated for 10 lesions in the following order: 1st lesion, posterior wall of the antrum; 2nd lesion, posterior wall/lesser curvature of the antrum; 3rd lesion, lesser curvature of the antrum; 4th lesion, greater curvature of the antrum; 5th lesion, anterior wall of the antrum; 6th lesion, greater curvature of the mid body; 7th lesion, posterior wall of
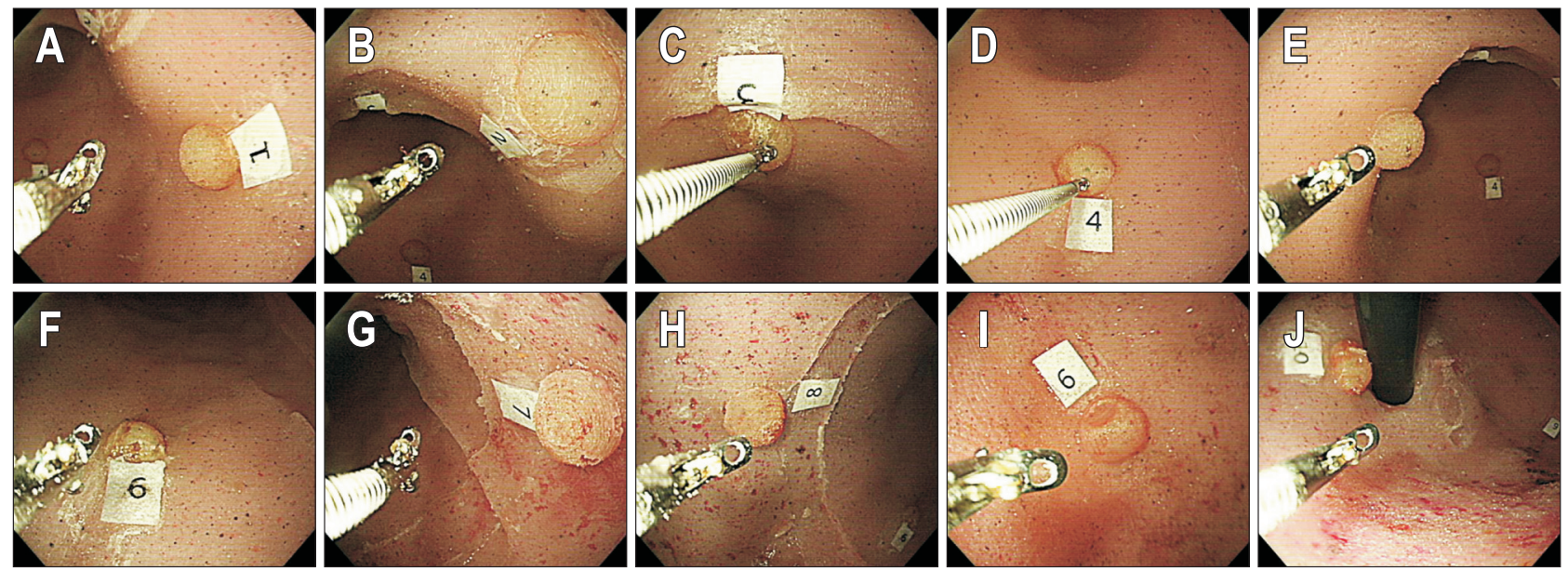

Fig. 2. Locations of the 10 individual lesions in the three-dimensional biopsy simulator. (A) 1st lesion, posterior wall of the antrum. (B) 2nd lesion, posterior wall/lesser curvature of the antrum. (C) 3rd lesion, lesser curvature of the antrum. (D) 4th lesion, greater curvature of the antrum. (E) 5th lesion, anterior wall of the antrum. (F) 6th lesion, greater curvature of the mid body. (G) 7th lesion, posterior wall of the mid body. (H) 8th lesion, anterior wall of the mid body. (I) 9th lesion, fundus. (J) 10th lesion, cardia. 
the mid body; 8th lesion, anterior wall of the mid body; 9th lesion, fundus; and 10th lesion, cardia (Fig. 2).

All participants performed five training sessions which were all video recorded in full. A timer was begun when the scope was inserted into the stomach and stopped when the scope returned to the gastroesophageal junction. The videos were reviewed after completion of the training, and the total completion time of each session, individual completion time for the 10 biopsy sites, and the approach time and biopsy time at each biopsy site were collected. The approach time was defined as the interval from the biopsy completion at the previous lesion to the steady positioning at the next biopsy site in the middle of the screen. The biopsy time was defined as the interval from the appearance of the biopsy forceps from the scope tip to the detachment of the forceps from the lesion with a piece of silicone tissue. The individual completion time was determined by adding the approach time and biopsy time for each lesion and the total completion time was the sum of the 10 individual completion times. The time required to insert the forceps was not counted as a procedure time.

\section{Questionnaire on simulator realism and training utility}

After completing five training sessions, all participants completed a self-administered questionnaire. The first 10 items of this questionnaire, which were used in a previous study, ${ }^{11}$ asked for opinions on the 3D biopsy simulator as follows: (1) endoscopic handling in the simulator is realistic (this item was not answered by the resident group); (2) the simulator is easy to handle; (3) training with the simulator is reasonable for endoscopic education; (4) working with the simulator improves my skills; (5-7) the following skills (introduction and positioning of the biopsy forceps, handling of the biopsy forceps, and interaction with the assistant) can be trained with the simulator; (8) the simulated biopsy procedure improves concentration during the training; (9) training with the simulator reduces the risk to patients; and (10) I recommend that the simulator be used in biopsy forceps training (this item was not answered by the resident group).
In addition, the difficulty levels and realism according to the locations in the simulator were estimated. The participants described their experiences in this regard using a 7-point Likert scale ( $1=$ strongly disagree to $7=$ strongly agree). The clinical fellows and staff determined the ranking of the difficulty level at each location in the simulator based on the experience of the real endoscopic biopsy at each location.

\section{Outcome measures}

The primary outcome measure was the total completion time with the newly developed biopsy simulator. We hypothesized that for the participants with no EGD experience, the completion time would rapidly decrease after the first trial. The secondary outcome measures were (1) the realism of the new simulator and (2) whether it could be recommended as an endoscopic biopsy training tool.

\section{Statistical analysis}

Continuous variables were reported as medians with IQRs and/or as means \pm standard deviations. Categorical variables were expressed as relative frequencies. Depending on the distribution, the two-sample t-test or the Mann-Whitney test was used to compare continuous variables and the chi-square or Fisher exact test (if the numbers were inadequate) to compare categorical variables. Correlations between the difficulty level and duration were analyzed using the Spearman rank test and linear regression. All p-values were two-sided, and p-values $<0.05$ were considered statistically significant. All statistical analyses were performed using the SPSS version 21.0 (IBM Corp., Armonk, NY, USA).

\section{RESULTS}

\section{Biopsy simulator outcomes}

The mean time (seconds) to complete the training procedure using the 3D biopsy simulator was $244.8 \pm 11.5$ in the resident group, $107.9 \pm 33.4$ in the first-year fellow group, $106.8 \pm 20.1$ in the second-year fellow group, and $103.8 \pm 19.2$ in the faculty

Table 1. Mean Duration to Complete the Procedure Using the 3D Biopsy Simulator according to the Experience Level of the Participant

\begin{tabular}{|c|c|c|c|c|}
\hline & Resident $(n=10)$ & First-year fellow $(n=6)$ & Second-year fellow $(n=5)$ & Faculty $(n=5)$ \\
\hline 1st session, s & $347.0 \pm 159.5^{*}$ & $143.8 \pm 33.1^{\dagger}$ & $127.2 \pm 21.5^{\ddagger}$ & $114.2 \pm 12.7$ \\
\hline 2nd session, s & $260.5 \pm 80.9^{*}$ & $113.7 \pm 29.5^{\dagger}$ & $108.6 \pm 23.6^{\ddagger}$ & $116.2 \pm 27.5$ \\
\hline 3rd session, s & $247.5 \pm 82.4^{*}$ & $103.8 \pm 30.3^{\dagger}$ & $101.6 \pm 17.7^{\ddagger}$ & $105.2 \pm 14.2$ \\
\hline 4th session, s & $199.6 \pm 72.1^{*}$ & $92.7 \pm 24.9^{\dagger}$ & $100.8 \pm 17.0^{\ddagger}$ & $92.2 \pm 14.1$ \\
\hline 5th session, $\mathrm{s}$ & $169.6 \pm 57.7^{*}$ & $85.7 \pm 22.0^{\dagger}$ & $96.0 \pm 7.8^{\ddagger}$ & $91.2 \pm 14.1$ \\
\hline Mean duration of the 5 trials, s & $244.8 \pm 11.5^{*}$ & $107.9 \pm 33.4^{\dagger}$ & $106.8 \pm 20.1^{\ddagger}$ & $103.8 \pm 19.2$ \\
\hline
\end{tabular}

Data are presented as mean \pm SD

3D, three-dimensional.

*p $<0.05$ versus the first-year fellow group, second-year fellow group, and faculty group; ${ }^{\dagger}$ p $>0.05$ versus the second-year fellow group and faculty group; ${ }^{\ddagger} \mathrm{p}>0.05$ versus the faculty group. 
group (Table 1). The mean duration of each trial was significantly longer in the resident group but not significantly different between the other three groups. As the trials were repeated, the completion times decreased significantly in all groups. The completion times in the fifth trial was significantly shorter than that in the first trial as follows: $169.6 \pm 57.7$ versus $347.0 \pm 159.5$ $(\mathrm{p}=0.007)$ in the resident group; $85.7 \pm 22.0$ versus $143.8 \pm 33.1$ $(\mathrm{p}=0.06)$ in the first-year fellow group; $96.0 \pm 7.8$ versus $127.2 \pm 21.5(\mathrm{p}=0.028)$ in the second-year fellow group; and $91.2 \pm 14.1$ versus $114.2 \pm 12.7,(\mathrm{p}=0.032)$ in the faculty group, respectively. The reduction was most prominent in the resident group (Fig. 3).

\section{Participant opinions on the 3D biopsy simulator}

Table 2 presents the mean scores recorded by the user questionnaires on the 3D biopsy simulator. The faculty group strongly agreed that endoscopic handling in the simulator was realistic and reasonable for endoscopic training $(6.2 \pm 0.8$ and

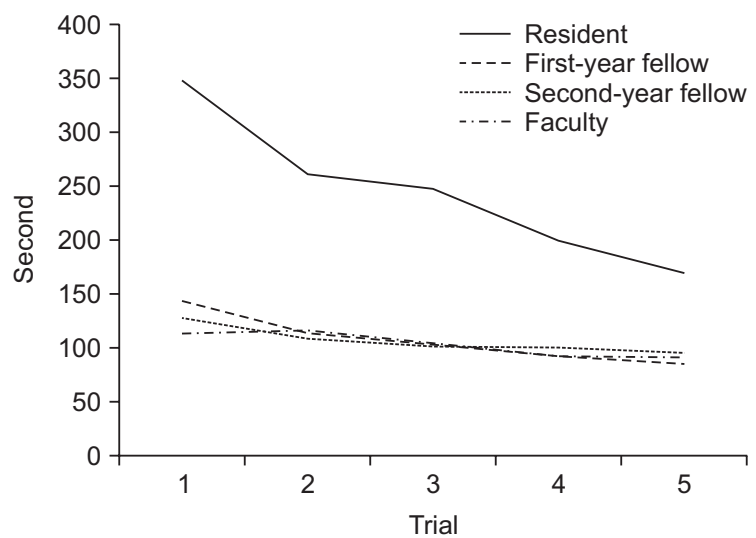

$6.4 \pm 0.9$, respectively). The simulator was considered significantly useful for skill improvement in the responses from the residents, first-year fellows, and second-year fellows. Notably, all of the participants felt that this simulator was effective as a training tool for each biopsy step. Significantly also, the experienced clinicians in the faculty group thought that this training could reduce the risk for patients and strongly endorsed this new simulator as a useful biopsy training method $(6.4 \pm 0.6$ and $7.0 \pm 0.0$, respectively).

\section{Simulator outcomes at each biopsy site}

Table 3 and Fig. 4 present the duration and difficulty scores for the 3D biopsy simulator according to the location of each lesion. The resident group spent significantly more time conducting the simulated biopsy at the 10 individual sites. In all of the groups that used the 3D biopsy simulator, the mean duration of tissue sampling from the lesion at the cardia (10th site) was longer than from any other lesions $(47.0 \pm 30.8$ in the resident group, $24.5 \pm 11.7$ in the first-year fellow group, $20.5 \pm 9.0$ in the second-year fellow group, and $21.9 \pm 8.4$ in the faculty group). The second most time-consuming site was the fundus in the resident group and mid body greater curvature in the other three groups. All groups experienced the highest difficulty level at the 10th lesion on the cardia, and it was notable that the three experienced groups conceded that this reflected the situation in the real stomach. Rank-order correlation analysis using the Spearman test indicated a significant relationship between the biopsy duration and difficulty level at each lesion (rho=0.720, $\mathrm{p}<0.001)$. Furthermore, the difficulty level at the 10 biopsy sites in the simulator coincided significantly with those in the real stomach according to the assessment by the experienced endoscopists (rho=0.753, $\mathrm{p}<0.001$ ).

Fig. 3. Completion time for each trial for all participant groups.

Table 2. Mean Scores for the 3D Biopsy Simulator Using a 7-Point Likert Scale and Based on the Experience Level of the Participants

\begin{tabular}{|c|c|c|c|c|}
\hline & $\begin{array}{l}\text { Resident } \\
(\mathrm{n}=10)\end{array}$ & $\begin{array}{l}\text { First-year fellow } \\
\qquad(\mathrm{n}=6)\end{array}$ & $\begin{array}{l}\text { Second-year fellow } \\
\qquad(\mathrm{n}=5)\end{array}$ & $\begin{array}{c}\text { Faculty } \\
(\mathrm{n}=5)\end{array}$ \\
\hline Endoscopic handling in the simulator is realistic. & NA & $5.8 \pm 1.0$ & $5.8 \pm 0.5$ & $6.2 \pm 0.8$ \\
\hline The simulator is easy to handle. & $5.9 \pm 1.3$ & $5.8 \pm 0.8$ & $5.2 \pm 0.8$ & $6.0 \pm 1.0$ \\
\hline Use of the simulator is reasonable for endoscopic training. & NA & $6.5 \pm 0.8$ & $6.0 \pm 1.2$ & $6.4 \pm 0.9$ \\
\hline Working with the simulator improves my skills. & $6.4 \pm 0.8$ & $5.8 \pm 1.0$ & $6.0 \pm 1.0$ & $2.0 \pm 2.2$ \\
\hline \multicolumn{5}{|l|}{ The following skills can be trained with the simulator: } \\
\hline Introduction and positioning of the biopsy forceps & $6.3 \pm 0.8$ & $6.0 \pm 0.9$ & $6.4 \pm 0.9$ & $6.6 \pm 0.9$ \\
\hline Handling of the biopsy forceps & $6.2 \pm 0.9$ & $6.3 \pm 0.8$ & $6.4 \pm 0.9$ & $5.8 \pm 0.8$ \\
\hline Interaction with the assistant & $6.0 \pm 1.3$ & $5.8 \pm 0.8$ & $6.6 \pm 0.6$ & $5.8 \pm 0.8$ \\
\hline Biopsy procedure improves concentration during simulator training. & $6.2 \pm 1.2$ & $5.8 \pm 0.8$ & $6.2 \pm 0.8$ & $6.2 \pm 0.8$ \\
\hline Training with the simulator reduces the risk for patients. & NA & $5.2 \pm 0.8$ & $5.6 \pm 1.3$ & $6.4 \pm 0.6$ \\
\hline I recommend that the simulator be used in biopsy training. & NA & $6.3 \pm 0.8$ & $6.4 \pm 0.9$ & $7.0 \pm 0.0$ \\
\hline
\end{tabular}

Data are presented as mean \pm SD.

Scale, 1 (strongly disagree) to 7 (strongly agree).

3D, three-dimensional; NA, not available. 
Table 3. Duration and Difficulty Scores for the 3D Biopsy Simulator according to the Location of the 10 Lesions and the Experience Level of the Participants

\begin{tabular}{|c|c|c|c|c|}
\hline Level of difficulty & Resident $(\mathrm{n}=10)$ & First-year fellow $(n=6)$ & Second-year fellow $(n=5)$ & Faculty $(n=5)$ \\
\hline \multicolumn{5}{|l|}{ 1st site (antrum PW) } \\
\hline Mean duration of 5 trials, $\mathrm{s}$ & $31.1 \pm 15.6^{*}$ & $12.2 \pm 5.2^{\dagger}$ & $12.3 \pm 2.4^{\ddagger}$ & $11 \pm 2.6$ \\
\hline Mean scores & $3.6 \pm 1.7$ & $2.5 \pm 1.2$ & $2.4 \pm 1.7$ & $1.8 \pm 0.8$ \\
\hline Rank of scores in the simulator/real stomach & 4/NA & $5 / 3$ & $4 / 4$ & $4 / 4$ \\
\hline \multicolumn{5}{|l|}{ 2nd site (antrum PW/LC) } \\
\hline Mean duration of 5 trials, $s$ & $15 \pm 9.3^{*}$ & $7.2 \pm 3.5^{\dagger}$ & $6.8 \pm 1.8^{\ddagger}$ & $7.5 \pm 3.3$ \\
\hline Mean scores & $1.8 \pm 0.8$ & $1.8 \pm 0.8$ & $1.6 \pm 0.9$ & $1.8 \pm 0.8$ \\
\hline Rank of scores in the simulator/real stomach & 10/NA & $7 / 7$ & $9 / 7$ & $4 / 5$ \\
\hline \multicolumn{5}{|l|}{ 3rd site (antrum LC) } \\
\hline Mean duration of 5 trials, $\mathrm{s}$ & $14.6 \pm 9.8^{*}$ & $6.8 \pm 2.8^{\dagger}$ & $8.2 \pm 1.5^{\ddagger}$ & $7.7 \pm 3.7$ \\
\hline Mean scores & $2.3 \pm 1.5$ & $1.8 \pm 1.0$ & $1.6 \pm 0.9$ & $1.4 \pm 0.6$ \\
\hline Rank of scores in the simulator/real stomach & 9/NA & $7 / 10$ & $9 / 10$ & $8 / 10$ \\
\hline \multicolumn{5}{|l|}{ 4th site (antrum GC) } \\
\hline Mean duration of 5 trials, $\mathrm{s}$ & $13.6 \pm 9.2^{\S}$ & $8.2 \pm 2.3^{\dagger}$ & $8 \pm 2.3^{\ddagger}$ & $7 \pm 1.8$ \\
\hline Mean scores & $2.4 \pm 1.1$ & $1.5 \pm 0.8$ & $2.2 \pm 1.1$ & $1.4 \pm 0.6$ \\
\hline Rank of scores in the simulator/real stomach & 8/NA & $10 / 8$ & $7 / 8$ & $8 / 8$ \\
\hline \multicolumn{5}{|l|}{ 5th site (antrum AW) } \\
\hline Mean duration of 5 trials, $\mathrm{s}$ & $13 \pm 9.0^{*}$ & $7.3 \pm 4.5^{\dagger}$ & $6.8 \pm 2.3^{\ddagger}$ & $6.5 \pm 2.5$ \\
\hline Mean scores & $2.6 \pm 1.0$ & $1.8 \pm 1.0$ & $2.4 \pm 0.9$ & $1.4 \pm 0.6$ \\
\hline Rank of scores in the simulator/real stomach & 7/NA & $7 / 7$ & $4 / 9$ & $8 / 9$ \\
\hline \multicolumn{5}{|l|}{ 6th site (mid body GC) } \\
\hline Mean duration of 5 trials, $\mathrm{s}$ & $28.6 \pm 16.7^{*}$ & $14.3 \pm 6.2^{\dagger}$ & $15.2 \pm 6.4^{\ddagger}$ & $13 \pm 4.3$ \\
\hline Mean scores & $4.1 \pm 1.7$ & $2.5 \pm 0.8$ & $4.4 \pm 2.0$ & $1.6 \pm 0.6$ \\
\hline Rank of scores in the simulator/real stomach & 3/NA & $5 / 9$ & $3 / 6$ & $7 / 7$ \\
\hline \multicolumn{5}{|l|}{ 7th site (mid body PW) } \\
\hline Mean duration of 5 trials, $\mathrm{s}$ & $18.1 \pm 7.1^{*}$ & $8.8 \pm 3.6^{\dagger}$ & $8.4 \pm 2.3^{\ddagger}$ & $8.8 \pm 2.2$ \\
\hline Mean scores & $3.4 \pm 1.0$ & $3.5 \pm 1.1$ & $2.2 \pm 1.3$ & $2.6 \pm 1.8$ \\
\hline Rank of scores in the simulator/real stomach & 5/NA & $3 / 2$ & $7 / 2$ & $3 / 2$ \\
\hline \multicolumn{5}{|l|}{ 8th site (mid body AW) } \\
\hline Mean duration of 5 trials, $\mathrm{s}$ & $18.4 \pm 10.9^{*}$ & $7.5 \pm 2.5^{\dagger}$ & $7.6 \pm 2.1^{\ddagger}$ & $8.5 \pm 2.8$ \\
\hline Mean scores & $3.1 \pm 0.7$ & $3.2 \pm 1.5$ & $2.4 \pm 1.1$ & $1.8 \pm 0.8$ \\
\hline Rank of scores in the simulator/real stomach & 6/NA & $4 / 6$ & $4 / 4$ & $4 / 6$ \\
\hline \multicolumn{5}{|l|}{ 9th site (fundus) } \\
\hline Mean duration of 5 trials, $s$ & $45.5 \pm 27.8^{*}$ & $11.2 \pm 5.3^{\dagger}$ & $13 \pm 4.1^{\ddagger}$ & $11.8 \pm 5.6$ \\
\hline Mean scores & $5.7 \pm 1.0$ & $4.5 \pm 1.8$ & $4.8 \pm 0.8$ & $4.8 \pm 2.6$ \\
\hline Rank of scores in the simulator/real stomach & 2/NA & $2 / 4$ & $2 / 3$ & $2 / 3$ \\
\hline \multicolumn{5}{|l|}{ 10th site (cardia) } \\
\hline Mean duration of 5 trials, $\mathrm{s}$ & $47 \pm 30.8^{*}$ & $24.5 \pm 11.7^{\dagger}$ & $20.5 \pm 9.0^{\ddagger}$ & $21.9 \pm 8.4$ \\
\hline Mean scores & $6.6 \pm 0.8$ & $6 \pm 1.3$ & $6.6 \pm 0.6$ & $6.4 \pm 0.9$ \\
\hline Rank of scores in the simulator/real stomach & $1 / \mathrm{NA}$ & $1 / 1$ & $1 / 1$ & $1 / 1$ \\
\hline
\end{tabular}

Data are presented as mean \pm SD.

3D, three-dimensional; PW, posterior wall; NA, not available; LC, lesser curvature; GC, greater curvature; AW, anterior wall.

${ }^{*} \mathrm{p}<0.05$ versus the first-year fellow group, second-year fellow group, and faculty group; ${ }^{\dagger} \mathrm{p}>0.05$ versus the second-year fellow group and faculty group; ${ }^{\ddagger} p>0.05$ versus the faculty group; ${ }^{\S} \mathrm{p}>0.05$ versus the first-year fellow group and second-year fellow group, $p<0.05$ versus the faculty group. 

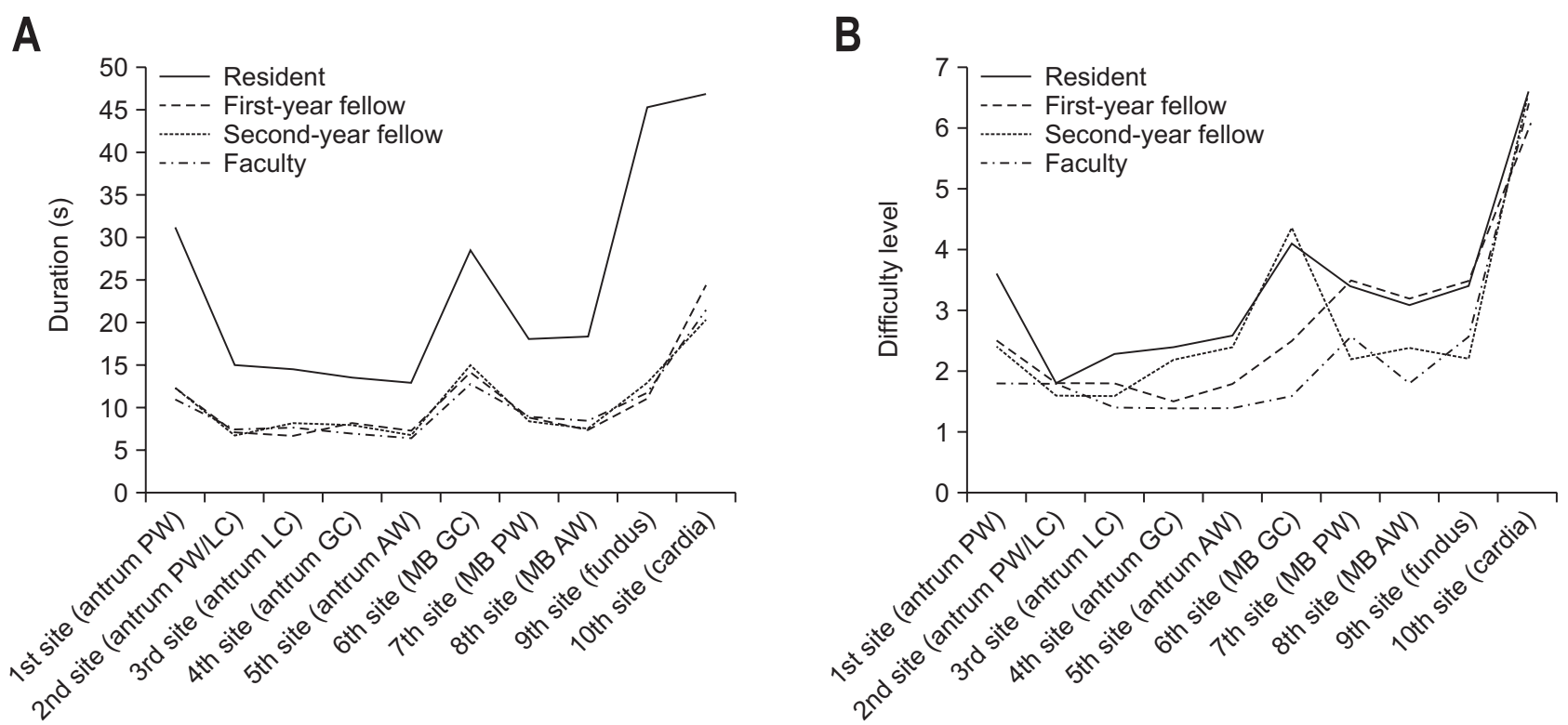

Fig. 4. Duration and difficulty levels for the three-dimensional biopsy simulator according to the location of the 10 lesions and the experience level of the participants. (A) Duration (s) and (B) difficulty levels (scale, 1=easiest to $7=$ most difficult).

$\mathrm{PW}$, posterior wall; LC, lesser curvature; GC, greater curvature; AW, anterior wall; MB, mid body.

Table 4. Mean Scores for the Level of Realism of the 3D Biopsy Simulator for Each Biopsy Site according to the Experience Level of the Participants Using a 7-Point Likert Scale

\begin{tabular}{|c|c|c|c|}
\hline & First-year fellow $(n=6)$ & Second-year fellow $(n=5)$ & Faculty $(n=5)$ \\
\hline 1st site (antrum PW) & $5.5 \pm 1.87$ & $6.2 \pm 1.3$ & $6.6 \pm 0.55$ \\
\hline 2nd site (antrum PW/LC) & $5.5 \pm 1.52$ & $6.8 \pm 0.45$ & $6.6 \pm 0.55$ \\
\hline 3rd site (antrum LC) & $6.0 \pm 1.27$ & $6.8 \pm 0.45$ & $6.4 \pm 0.55$ \\
\hline 4th site (antrum GC) & $6.3 \pm 1.03$ & $6.0 \pm 1.73$ & $6.6 \pm 0.55$ \\
\hline 5th site (antrum AW) & $6.3 \pm 0.82$ & $6.4 \pm 0.55$ & $6.2 \pm 0.84$ \\
\hline 6th site (mid body GC) & $5.8 \pm 1.17$ & $5.4 \pm 1.52$ & $6.4 \pm 0.89$ \\
\hline 7th site (mid body PW) & $5.3 \pm 2.07$ & $5.8 \pm 0.84$ & $6.4 \pm 0.89$ \\
\hline 8th site (mid body AW) & $6.2 \pm 0.98$ & $6.2 \pm 1.3$ & $6.4 \pm 0.89$ \\
\hline 9th site (fundus) & $6.8 \pm 0.41$ & $5.6 \pm 1.67$ & $6.6 \pm 0.55$ \\
\hline 10th site (cardia) & $7.0 \pm 0.0$ & $5.4 \pm 1.67$ & $5.0 \pm 2.83$ \\
\hline
\end{tabular}

Data are presented as mean \pm SD.

Scale, 1 (strongly disagree) to 7 (strongly agree).

3D, three-dimensional; PW, posterior wall; LC, lesser curvature; GC, greater curvature; AW, anterior wall.

\section{Participants' opinions on the realism of the 3D biopsy simulator}

The experienced endoscopists also estimated the realism level of the 3D biopsy simulator for the 10 biopsy sites using the 7 -point Likert scale (Table 4). They assigned $>5$ points to all sites on average, indicating agreement that the 3D biopsy simulator was realistic in terms of the location of each lesion. There were no significant differences between the Likert scores of the three groups. For the 10th lesion on the cardia, one secondyear fellow and two faculty members were not satisfied with the realism due to a lower elasticity in the simulator which made it difficult to approach the lesion.

\section{DISCUSSION}

We assessed the efficacy and realism of a novel 3D endoscopic biopsy simulator, manufactured using a 3D printer and silicone, through a system of trials by participants with differing levels of experience, including novices. Not surprisingly, the biopsy completion times using this simulator differed in accordance with the degree of participant experience with an endoscopic examination: the nonexperienced group (resident group) required a longer time to complete a training session than the other groups. However, the completion time became shorter as the participants repeated the training sessions and was most prominently reduced for the nonexperienced subjects. The ex- 
pert group strongly agreed that the simulator was realistic in terms of endoscopic handling and reasonable for endoscopic training. Meanwhile, the trainee groups considered that this simulator was a useful tool for improving their biopsy skills. In terms of the location of each lesion, the experienced endoscopists reported that the difficulty levels at the 10 biopsy sites in the simulator coincided well with those in the real stomach. Therefore, our new system can successfully simulate the conditions in the real stomach and helped to improve the endoscopic biopsy skills of trainee clinicians without any risks to patients.

Three-dimensional printing is an advanced manufacturing technology which enables a 3D structure to be produced from a computer-aided design and is becoming more and more sophisticated over time. ${ }^{12}$ In recent decades, 3D printing has been used in many nonmedical fields with innovative results and this technology is thus generating great interest in medicine. In anatomical education and surgical training in particular, 3D printing has shown promising outcomes and new applications. ${ }^{5,6,13-17}$ In the current study, we employed 3D printing to synthesize a endoscopic biopsy simulator for the stomach, decrease the production costs associated with such an endeavor, improve the realism of the simulator, and of course eliminate the risk associated with using patient volunteers. Although virtual simulators and tissue-based simulators have been developed in the past and have shown efficacy in previous studies, their availability is limited owing to their high cost and difficulties in terms of management and accessibility. ${ }^{3,18}$ With our new simulator, however, biopsy techniques in a realistic stomach-model system can be practiced at any time and there are no risks to any patients. This simulator is $55 \mathrm{~cm}$ in length and weighs $3.3 \mathrm{~kg}$, and is thus very portable. We used three $3 \mathrm{D}$ printers in our current study to produce the stomach models, but a single 3D printer could be used which would incur a cost of only US \$2000 (3D printer, Clone K300, US \$1760; material costs, including the 3D filament, acrylic plate, and silicone, US \$230). Hence, our 3D biopsy simulator enhances trainee accessibility, realism in terms of stomach anatomy, and more cost-effectiveness.

Some limitations of our novel 3D biopsy simulator are noteworthy. First, inflation and deflation of the simulator is possible to some degree because of the elasticity of silicone. However, gastric peristalsis and the movement of the stomach due to the heartbeat and respiration are not reproduced; thus, targeting a lesion in our simulator will be easier than in a real stomach. Second, we could not reproduce the same level of elasticity as a real stomach. As a result of this, the scores relating to the realism of the lesion on the cardia were rated lower by the more experienced study participants. Third, the completion time for the 6th lesion at the greater curvature of the mid body was longer than expected, and some participants recorded high difficulty scores at this site. We attributed this to the overlying glue, which made the lesion more difficult to sample. Finally, there was a possible bias in evaluating the simulator performance using questionnaires. The developer of this simulator did not participate in this study but is a more senior faculty member than our faculty group subjects. This could have influenced the evaluations by these participants. Nevertheless, our 3D-printed simulator has shown great promise and can form the basis of further studies on enhancing endoscopic training tools using the latest cutting-edge technologies.

In conclusion, an endoscopic biopsy simulator created using a $3 \mathrm{D}$ printer is an effective and realistic method for improving biopsy skills without any risk to patients. This simulator provides a realistic model of a real stomach in which trainees can gain experience with endoscopic manipulation, especially for biopsy. The simulator can be produced at a relatively low cost, can be used by a large number of trainees repeatedly, and can raise their biopsy skills to an acceptable level before performing an endoscopy in actual patients.

\section{CONFLICTS OF INTEREST}

No potential conflict of interest relevant to this article was reported.

\section{ACKNOWLEDGEMENTS}

This study was supported by both the Korean College of Helicobacter and Upper Gastrointestinal Research Foundation grant and a grant (2016-671) from the Asan Institute for Life Sciences, Asan Medical Center, Seoul, Korea.

\section{REFERENCES}

1. Bisschops R, Wilmer A, Tack J. A survey on gastroenterology training in Europe. Gut 2002;50:724-729.

2. Bini EJ, Firoozi B, Choung RJ, Ali EM, Osman M, Weinshel EH. Systematic evaluation of complications related to endoscopy in a training setting: a prospective 30-day outcomes study. Gastrointest Endosc 2003;57:8-16.

3. Cohen J, Thompson CC. The next generation of endoscopic simulation. Am J Gastroenterol 2013;108:1036-1039.

4. Ahn JY, Lee JS, Lee GH, et al. The efficacy of a newly designed, easy-to-manufacture training simulator for endoscopic biopsy of the stomach. Gut Liver 2016;10:764-772.

5. Wurm G, Tomancok B, Pogady P, Holl K, Trenkler J. Cerebrovascular stereolithographic biomodeling for aneurysm surgery: technical note. J Neurosurg 2004;100:139-145.

6. AbouHashem Y, Dayal M, Savanah S, Štrkalj G. The application of 3D printing in anatomy education. Med Educ Online 2015;20:29847.

7. Naftulin JS, Kimchi EY, Cash SS. Streamlined, inexpensive 3D printing of the brain and skull. PLoS One 2015;10:e0136198.

8. Tack P, Victor J, Gemmel P, Annemans L. 3D-printing techniques in a medical setting: a systematic literature review. Biomed Eng 
Online 2016;15:115.

9. Zerr J, Chatzinoff Y, Chopra R, Estrera K, Chhabra A. Threedimensional printing for preoperative planning of total hip arthroplasty revision: case report. Skeletal Radiol 2016;45:1431-1435.

10. Fedorov A, Beichel R, Kalpathy-Cramer J, et al. 3D Slicer as an image computing platform for the quantitative imaging network. Magn Reson Imaging 2012;30:1323-1341.

11. Schneider AR, Schepp W. Do it yourself: building an ERCP training system within 30 minutes (with videos). Gastrointest Endosc 2014;79:828-832.

12. Peltola SM, Melchels FP, Grijpma DW, Kellomäki M. A review of rapid prototyping techniques for tissue engineering purposes. Ann Med 2008;40:268-280.

13. Barber SR, Kozin ED, Dedmon M, et al. 3D-printed pediatric endoscopic ear surgery simulator for surgical training. Int J Pediatr Otorhinolaryngol 2016;90:113-118.
14. Jones DB, Sung R, Weinberg C, Korelitz T, Andrews R. Threedimensional modeling may improve surgical education and clinical practice. Surg Innov 2016;23:189-195.

15. Soon DS, Chae MP, Pilgrim CH, Rozen WM, Spychal RT, HunterSmith DJ. 3D haptic modelling for preoperative planning of hepatic resection: a systematic review. Ann Med Surg (Lond) 2016;10:1-7.

16. VanKoevering KK, Hollister SJ, Green GE. Advances in 3-dimensional printing in otolaryngology: a review. JAMA Otolaryngol Head Neck Surg 2017;143:178-183.

17. Baskaran V, Štrkalj G, Štrkalj M, Di Ieva A. Current applications and future perspectives of the use of $3 \mathrm{D}$ printing in anatomical training and neurosurgery. Front Neuroanat 2016;10:69.

18. Qiao W, Bai Y, Lv R, et al. The effect of virtual endoscopy simulator training on novices: a systematic review. PLoS One 2014; 9:e89224. 